\title{
EFICIÊNCIA DO TERBUFÓS E DO CARBOFURAN \\ NO CONTROLE DA BROCA DA BANANEIRA, Cosmopolites sordidus, GERMAR (COLEOPTERA: CURCULIONIDAE)
}

\author{
FLÁVIA RABELO BARBOSA * \\ EDUARDO ALVES DE SOUZA ** \\ WELLINGTON ANTONIO MOREIRA *** \\ JOSÉ ADALBERTO DE ALENCAR **** \\ FRANCISCA NEMAURA PEDEROSA HAJI * \\ CHERRE SADE BEZERRA DA SILVA **
}

\begin{abstract}
Avaliou-se a eficiência de dois inseticidas granulados, terbufós e carbofuran em diferentes dosagens, no controle da broca da bananeira Cosmopolites sordidus. Também avaliou-se o efeito dos equipamentos de aplicação Swissmex (S) e Matraca (M) na eficiência do terbufós. O experimento foi instalado em plantio comercial, no município de Petrolina-PE (Brasil), em blocos ao acaso com seis tratamentos e cinco repetições. Os tratamentos ( $\mathrm{g}$ ingrediente ativo/cova - equipamento aplicação) foram: 1. terbufós $150 \mathrm{G}(1,95) \mathrm{S}$; 2. terbufós $150 \mathrm{G}$ $(3,00)$ S; 3. terbufós $50 \mathrm{G}(3,00) \mathrm{S} ; 4$. terbufós $50 \mathrm{G}$ $(3,00) \mathrm{M} ; 5$. carbofuran $50 \mathrm{G}(4 \mathrm{~g}) \mathrm{M}$ e 6 . testemunha (não-tratada). Nos tratamentos 1, 2 e 3 o inseticida foi aplicado com o equipamento Swissmex (S) e nos tratamentos 4 e 5 com Matraca (M). Foram utilizadas 30 plantas da cv. Pacovan por tratamento, sendo avaliado o número de insetos antes da aplicação. Mensurou-se o efeito dos tratamentos sobre a praga aos 90 e 120 dias após a aplicação, com base nas galerias decorrentes da infestação da broca. Os dados foram estatisticamente analisados e as médias comparadas pelo teste de Tukey a $5 \%$. Dados do coeficiente de infestação dos rizomas foram transformados em $\sqrt{x+0,5}$. Calculou-se a eficiência média dos tratamentos pela fórmula de Abbott. Os produtos terbufós e carbofuran, nas dosagens testadas, mostraram-se eficientes no controle da broca da bananeira, contudo não se constatou diferença significativa entre os tratamentos químicos, nem entre os equipamentos de aplicação. As percentagens de eficiência dos produtos testados variaram de 96,7 a $100 \%$.
\end{abstract}

PALAVRAS-CHAVE: TERBUFÓS; CARBOFURAN; INSETICIDAS; MOLEQUE-DA-BANANEIRA; Musa spp.

* Pesquisador, Doutora em Entomologia, Embrapa Semi-Árido, Petrolina, PE (e-mail: flavia@cpatsa.embrapa.br).

** Estagiário Embrapa Semi-Árido, Petrolina, PE.

*** Pesquisador, Doutor em Fitopatologia, Embrapa Semi-Árido, Petrolina, PE.

**** Pesquisador, Mestre em Entomologia, Embrapa Semi-Árido, Petrolina, PE. 


\section{INTRODUÇÃO}

A bananicultura constitui atividade agrícola de interesse no Brasil, assumindo elevada importância econômica e social. Responde pela produção de alimento básico para as populações carentes de diversos países, estando também presente na mesa de todas as camadas sociais da população. $O$ Brasil é o segundo maior produtor e o maior consumidor mundial de banana (FAO, 2001 citado por FERREIRA, CORDEIRO e MATOS, 2003). Em 2003, a exportação dessa fruta ultrapassou a cifra de US $\$ 30$ milhões, reprsentando quase $9 \%$ do volume auferido com as 25 principais frutas frescas exportadas pelo País. Trata-se da quinta fruta mais comercializada em faturamento e a primeira em peso (Anuário Brasileiro da Fruticultura, 2004). As regiões Nordeste e Sudeste exercem grande influência na produção nacional de banana, respondendo por aproximadamente $80 \%$ do volume colhido e da área plantada (SILVA e CORDEIRO, 2000).

Embora muitos insetos encontrem-se associados à bananeira, poucos são os que causam danos significativos à plantação. Das pragas que freqüentemente exigem medidas para o seu controle destaca-se a broca da bananeira, Cosmopolites sordidus (Coleoptera: Curculionidae). Trata-se de praga severa, que atinge praticamente todas as regiões em que se cultiva a banana. Altas populações de broca são responsáveis por quedas de safras de até $80 \%$, em função da redução da colheita e da qualidade do produto. Além disso, seu ataque pode facilitar a penetração de outros insetos, nematóides e microorganismos (FANCELLI e MESQUITA, 2000). Os danos que evidenciam o ataque da praga são causados pelas larvas, que constroem galerias no rizoma. Plantas severamente infestadas têm 0 sistema radicular comprometido, apresentando sintomas iniciais de amarelecimento, com posterior secamento das folhas e morte do broto. Em plantas jovens pode ocorrer destruição da gema apical. Verifica-se, também, maior suscetibilidade ao tombamento, pela ação do vento, principalmente em plantas com cacho (FANCELLI, 1997).

Para o controle da broca-da-bananeira, o tratamento químico tem sido utilizado com maior freqüência pelos produtores. A aplicação dos inseticidas sistêmicos registrados para a cultura da banana (ANVISA, 2004), como o terbufós (organofosforado) e o carbofuran (carbamato), tem sido recomendada por apresentarem também ação nematicida (COSTA, 2000; FANCELLI e MESQUITA, 2000).

Este trabalho teve como objetivo avaliar a eficiência do terbufós e do carbofuran no controle de C. sordidus, bem como de dois tipos de equipamento para aplicação do terbufós.

\section{MATERIAL E MÉTODOS}

O trabalho foi conduzido em pomar comercial, com dois anos de idade, pertencente ao Projeto de Irrigação Senador Nilo Coelho, no município de Petrolina-PE. Para a aplicação do terbufós $150 \mathrm{G}$ utilizou-se como equipamento o Swissmex (S), enquanto que o carbofuran $50 \mathrm{G}$ foi aplicado com Matraca (M). Para avaliar o efeito do tipo de equipamento na eficiência do produto utilizou-se swissmex e matraca para o terbufós 50G. Os tratamentos, concentrações, formulações, dosagens dos inseticidas (em gramas de ingrediente ativo por cova) e equipamentos de aplicação foram: 1. terbufós $150 \mathrm{G}(1,95)$ S; 2. terbufós $150 \mathrm{G}(3,00) \mathrm{S} ; 3$. terbufós $50 \mathrm{G}(3,00) \mathrm{S} ; 4$. terbufós $50 \mathrm{G}(3,00) \mathrm{M} ; 5$. carbofuran $50 \mathrm{G}$ (4 g) M e 6. testemunha (não-tratada). Foi realizada apenas uma aplicação dos produtos na cova. Para a avaliação prévia da infestação do bananal por $C$. sordidus, iscas tipo queijo foram utilizadas (FANCELLI e MESQUITA, 2000).

Adotou-se o delineamento experimental de blocos ao acaso com cinco repetições, sendo a unidade experimental constituída por seis plantas da cultivar Pacovan, em plena produção. Mensurouse o efeito dos tratamentos sobre a praga pelo método de MESQUITA (1985), citado por FANCELLI (1997). Esse, consiste em corte transversal do rizoma na altura de seu máximo diâmetro e observação de galerias decorrentes do ataque da broca (Quadro 1). Subdividiu-se a área total do rizoma em quatro 
subáreas (cada uma com o valor de $25 \%$ ), sendo a percentagem de infestação por planta dada pela soma dos quatro valores. Realizaram-se amostragens do número de rizomas infestados por $C$. sordidus aos 90 e 120 dias. Para análise de variância, os dados referentes ao coeficiente de infestação dos rizomas foram transformados em $\sqrt{x+0,5}$. Compararam-se as médias pelo teste de Tukey, ao nível de $5 \%$ de probabilidade, sendo a eficiência dos produtos mensurada pela fórmula de ABBOTT (1925).

\section{QUADRO 1 - ESCALA DE NOTAS PARA AVALIAÇÃO DO COEFICIENTE DE INFESTAÇÃO DE RIZOMAS DE BANANEIRA ATACADOS POR Cosmopolites sordidus ${ }^{1}$}

\begin{tabular}{|l|l|}
\hline Nota & CritØrio de Notas \\
\hline 0 & AusĆ̈cia de galerias \\
5 & Presen a de tra os de galerias \\
10 & Nota intermedi/fía entre 5 e 20 \\
20 & Presen a de galerias em um quarto da periferia do rizoma \\
30 & Nota intermedi/fria entre 20 e 40 \\
40 & Presen a de galerias na metade da periferia do rizoma \\
60 & Presen a de galerias em trđs quartos da periferia do rizoma \\
100 & Presen a de galerias em toda a periferia do rizoma \\
\hline
\end{tabular}

${ }^{1}$ MESQUITA (1985), citado por FANCELLI (1997).

As possíveis alterações morfofisiológicas da planta, que pudessem ser caracterizadas por efeito tóxico dos produtos foram avaliadas segundo a escala de notas da EWRC (REPORT..., 1964) (Quadro 2).

\section{QUADRO 2 - ESCALA DE NOTAS PARA AVALIAÇÃO DA FITOTOXICIDADE DOS INSETICIDAS PARA A BANANEIRA ${ }^{1}$}

\begin{tabular}{|l|l|}
\hline Nota & Fitotoxicidade \\
\hline 1 & nula \\
2 & muito leve \\
3 & leve \\
4 & regular \\
5 & mødia \\
6 & quase forte \\
7 & forte \\
8 & muito forte \\
9 & destrui a o total \\
\hline
\end{tabular}

${ }^{1}$ REPORT... (1964).

\section{RESULTADOS E DISCUSSÃO}

$\mathrm{Na}$ avaliação prévia da infestação do bananal por $C$. sordidus constatou-se, em média, 6,1 insetos por isca, superando o nível de controle preconizado para essa praga que é de 2 a 5 insetos/ isca (FANCELLI e MESQUITA, 2000).

Aos 90 e 120 dias após a aplicação, os produtos terbufós e carbofuran (nas dosagens testadas) causaram redução significativa no coeficiente de infestação dos rizomas pela broca da bananeira. 
Contudo, não foram observadas diferenças significativas nas percentagens de eficiência média entre os produtos e as dosagens testadas (Tabela 1). De acordo com VILARDEBO, citado por SAMPAIO et al. (1982), a produtividade do bananal é diretamente proporcional ao coeficiente de infestação dos rizomas.

As percentagens de eficiência média dos tratamentos no controle da praga até 120 dias após a aplicação foram de $97,59 \%$ para terbufós $150 \mathrm{G}$ (1,95 e 3,0 g i.a./cova) e 96,79\% para terbufós $50 \mathrm{G}$

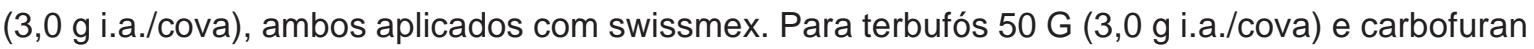
$50 \mathrm{G}$ (4,0 g i.a./cova), aplicados com matraca a média foi de 100\%. A eficiência do carbofuran no controle dessa praga foi também constatada por FAZOLIN, PEREIRA e WILCKEN (1990) e SAMPAIO et al. (1982).

Com relação ao efeito do equipamento na eficiência do terbufós $50 \mathrm{G}$, embora não tenha sido constatada diferença significativa, observaram-se coeficientes de infestação da broca de 1,67 e 0,0 para swissmex e matraca, respectivamente (Tabela 1).

\section{TABELA 1 - EFEITO DOS TRATAMENTOS NA INFESTAÇÃO DE RIZOMAS DE BANANEIRA POR Cosmopolites sordidus, AOS 90 E 120 DIAS APÓS A APLICAÇÃO DOS INSETICIDAS - PETROLINA (PE) 2004}

\begin{tabular}{|c|c|c|c|c|c|}
\hline \multirow[t]{2}{*}{ Tratamento $^{1}$} & \multirow{2}{*}{$\begin{array}{c}\text { Dosagem } \\
\text { (g i.a. cova) }\end{array}$} & \multicolumn{3}{|c|}{$\begin{array}{l}\text { Coeficiente de infesta }{ }^{\underline{a}} 0^{2} \\
\text { (dias/nota) }\end{array}$} & \multirow{2}{*}{$\begin{array}{c}\text { Eficiđ̄́cia } \\
\text { MØdia }^{3} \\
(\%)\end{array}$} \\
\hline & & 90 & 120 & MØlia & \\
\hline Testemunha & - & 59,17 & 44,17 & $51,67 \mathrm{a}$ & - \\
\hline Terbuf s $150 \mathrm{G}-\mathrm{S}$ & 1,95 & 2,50 & 0,00 & $1,25 b$ & 97,59 \\
\hline Terbuf s $150 \mathrm{G}-\mathrm{S}$ & 3,00 & 0,00 & 2,50 & $1,25 \mathrm{~b}$ & 97,59 \\
\hline Terbuf s $50 \mathrm{G} \quad \mathrm{M}$ & 3,00 & 0,00 & 3,34 & $0,00 \mathrm{~b}$ & 100,00 \\
\hline Terbuf s $150 \mathrm{G}-\mathrm{S}$ & 3,00 & 0,00 & 0,00 & $1,25 \mathrm{~b}$ & 97,59 \\
\hline Carbofuran $50 \mathrm{G} \quad \mathrm{M}$ & 4,00 & 0,00 & 0,00 & $0,00 \mathrm{~b}$ & 100,00 \\
\hline
\end{tabular}

${ }^{1}$ Tipo de equipamento utilizado na aplicação do inseticida: $S=S$ Swissmex; $M=$ Matraca.

${ }^{2} 0$ = ausência de galerias; 5 = presença de traços de galerias; 10 = infestação entre 5 e $20 ; 20$ = presença de galerias em um quarto da periferia do rizoma; 30 = nota intermediária entre 20 e $40 ; 40$ = presença de galerias na metade da periferia do rizoma; 60 = presença de galerias em três quartos da periferia do rizoma; $100=$ presença de galerias em toda a periferia do rizoma (MESQUITA, 1985, citado por FANCELLI, 1997). Médias seguidas pela mesma letra, na coluna, não diferem entre si pelo teste de Tukey ao nível de $5 \%$ de probabilidade (valores não-transformados).

${ }^{3}$ Calculada pela fórmula de ABBOTT (1925).

No decorrer do período experimental, ou seja, até a realização da última avaliação (120 dias após a aplicação dos produtos) não foram observadas alterações nas plantas, como clorose e necrose, decorrentes de fitotoxicidade. Tal fato confere a nota 1 aos tratamentos utilizados conforme a escala desenvolvida pelo EWRC (REPORT..., 1964) (Quadro 2).

\section{CONCLUSÃO}

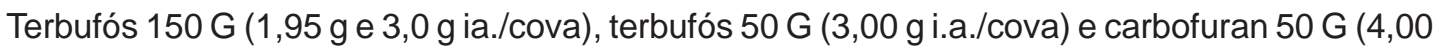
$\mathrm{g}$ i.a./cova) foram eficientes no controle da broca da bananeira, Cosmopolites sordidus.

Não foi constatada diferença significativa na eficiência do terbufós $50 \mathrm{G}$, quando aplicado por Swissmex ou Matraca. 


\begin{abstract}
EFFICIENCY OF TERBUFOS AND CARBOFURAN ON THE CONTROL OF BANANA WEEVIL BORER, Cosmopolites sordidus, GERMAR (COLEOPTERA: CURCULIONIDAE)

The efficiency of two granulated insecticides, terbufos and carbofuran in different dosages in the control of banana weevil borer Cosmopolites sordidus was evaluated. The application equipments Swissmex (S) and Matraca (M) effects in the efficiency of terbufos were also studied. The experiment was held in commercial plantation, in the district of Petrolina-PE (Brazil) in randomized blocks with six treatments and five repetitions. The treatments $(\mathrm{g}$ active ingredient/ planting hole - application equipment) were: 1 . terbufos $150 \mathrm{G}$ (1.95) S; 2. terbufos $150 \mathrm{G}$ (3.00) S; 3. terbufos $50 \mathrm{G}(3.00)$ $\mathrm{S} ; 4$. terbufos $50 \mathrm{G}(3.00) \mathrm{M}$; 5. carbofuran $50 \mathrm{G}(4 \mathrm{~g}) \mathrm{M}$ and 6. control (non treated). In the treatments 1,2 and 3 the insecticide was applied with the equipment Swissmex (S) and in the treatments 4 and 5 with Matraca (M). Thirty (30) cV. Pacovan plants were utilized per treatment, being evaluated the number of insects before application. The effect of the treatments on the pest was evaluated at 90 and 120 days after application, with basis in the galleries from borer infestation. The data were statistically analyzed and the means compared by Tukey test at $5 \%$. Data of infestation coefficient of the rhizomes were transformed into $\sqrt{x+0.5}$. The mean efficiency was calculated using the Abbot formula. The products terbufos and carbofuran, in tested dosages showed to be effective in the control ob banana weevil borer, although, there weren't significant differences between the chemical treatments neither between the application equipments. The efficiency percentages of the tested products varied from 96.7 to $100 \%$.
\end{abstract}

KEY-WORDS: TERBUFOS; CARBOFURAN; INSECTICIDES; BANANA WEEVIL BORER; Musa spp.

\title{
REFERÊNCIAS
}

1 ABBOTT, W.S. A method of computing the effectiveness of an insecticide. Journal of Economic Entomology, v.18, p.265-267, 1925

2 ANUÁRIO BRASILEIRO DA FRUTICULTURA. Gazeta Santa Cruz, Santa Cruz do Sul, p.30-33, 2004.

3 ANVISA. Sistema de informações sobre agrotóxicos. Disponível em: http://www4.anvisa.gov.br/agrosia/ asp/default.asp. Acesso em: 15 jan. 2004.

COSTA, D. da C. Doenças causadas por nematóides. In: CORDEIRO, Z.J.M. (Ed.). Banana: fitossanidade. Brasília: Embrapa Comunicação para Transferência de Tecnologia, 2000. p.65-77 (Frutas do Brasil, 8).

FANCELLI, M. Pragas. In: ALVES, E.J. (Ed.). A cultura da banana: aspectos técnicos, socioeconômicos e agroindustriais. Brasília: Embrapa-SPI, 1997. p.409-451.

FANCELLI, M.; MESQUITA, A.L.M. Pragas. In: CORDEIRO, Z.J.M. (Ed.). Banana: fitossanidade. Brasília: Embrapa Comunicação para Transferência de Tecnologia, 2000. p.21-35 (Frutas do Brasil, 8).

FAZOLIN, M.; PEREIRA, L.V.; WILCKEN, C.F. Influência de três inseticidas sobre o controle e atratividade de Cosmopolites sordidus (Germar, 1824) (Coleoptera, Curculionidae) em iscas tipo queijo, no município de Rio Branco (AC). Anais da Sociedade Entomológica do Brasil, Brasília, v. 19, n. 1, p. 181-189, 1990.

FERREIRA, D.M.V.; CORDEIRO, Z.J.M.; MATOS, A.P. de. Sistema de pré-aviso para o controle da sigatoka-amarela da bananeira no Recôncavo Baiano. Revista Brasileira de Fruticultura, Jaboticabal, v.25, n. 3, p. 429-431, 2003.

9 REPORT of the third and fourth meetings of the EWRC. Committee on Methods. Weed Research (Notes and News), Oxford, v.4, p.88, 1964.

10 SAMPAIO, A.S.; MYAZAKI, I.; SUPLICY FILHO, N.; OLIVEIRA, D.A. "Broca da bananeira" - Cosmopolites sordidus (Germar, 1824) (Coleoptera; Curculionidae) resistente ao aldrin e seu controle com inseticidas sistêmicos aplicados no solo. O Biológico, v. 48, p. 91-98, 1982.

11 SILVA, J.R. da; CORDEIRO, Z.J.M. Fitossanidade na exportação de banana. In: CORDEIRO, Z.J.M. (Ed.). Banana: fitossanidade. Brasília: Embrapa Comunicação para Transferência de Tecnologia, 2000. p.9-14 (Frutas do Brasil, 8). 